\title{
BMJ Open Question design in nurse-led and GP-led telephone triage for same-day appointment requests: a comparative investigation
}

\author{
Jamie Murdoch, ${ }^{1}$ Rebecca Barnes, ${ }^{2}$ Jillian Pooler, ${ }^{3}$ Val Lattimer, ${ }^{1}$ Emily Fletcher, ${ }^{3}$ \\ John L Campbell ${ }^{3}$
}

To cite: Murdoch J, Barnes R, Pooler J, et al. Question design in nurse-led and GP-led telephone triage for same-day appointment requests: a comparative investigation. BMJ Open 2014:4:e004515.

doi:10.1136/bmjopen-2013004515

- Prepublication history for this paper is available online. To view these files please visit the journal online (http://dx.doi.org/10.1136/ bmjopen-2013-004515).

Received 20 November 2013 Revised 28 January 2014 Accepted 10 February 2014

CrossMark

${ }^{1}$ Faculty of Medicine and Health Sciences, School of Nursing Sciences, Edith Cavell Building, University of East Anglia, Norwich Research Park, Norwich, UK ${ }^{2}$ Centre for Academic Primary Care, School for Social and Community Medicine, Bristol, UK

${ }^{3}$ Primary Care Research Group, University of Exeter Medical School, University of Exeter, Exeter, UK

Correspondence to Dr Jamie Murdoch; Jamie.murdoch@uea.ac.uk

\section{ABSTRACT}

Objective: To compare doctors' and nurses' communication with patients in primary care telephone triage consultations.

Design: Qualitative comparative study of content and form of questions in 51 telephone triage encounters between practitioners (general practitioners $(\mathrm{GPs})=29$; nurses=22) and patients requesting a same-day appointment in primary care. Audio-recordings of nurse-led calls were synchronised with video recordings of nurse's use of computer decision support software (CDSS) during triage.

Setting: 2 GP practices in Devon and Warwickshire, UK.

Participants: 4 GPs and 29 patients; and 4 nurses and 22 patients requesting a same-day face-to-face appointment with a GP.

Main outcome measure: Form and content of practitioner-initiated questions and patient responses during clinical assessment.

Results: A total of 484 question-response sequences were coded (160 GP; 324 N). Despite average call lengths being similar ( $\mathrm{GP}=4 \mathrm{~min}, 37 \mathrm{~s},(\mathrm{SD}=1 \mathrm{~min}$, $26 \mathrm{~s}) ; \mathrm{N}=4 \mathrm{~min}, 39 \mathrm{~s},(\mathrm{SD}=2 \mathrm{~min}, 22 \mathrm{~s}))$, GPs and nurses differed in the average number ( $\mathrm{GP}=5.51$,

$(S D=4.66) ; N=14.72,(S D=6.42)$ ), content and form of questions asked. A higher frequency of questioning in nurse-led triage was found to be due to nurses' use of CDSS to guide telephone triage. $89 \%$ of nurse questions were oriented to asking patients about their reported symptoms or to wider-information gathering, compared to $54 \%$ of GP questions. $43 \%$ of GP questions involved eliciting patient concerns or expectations, and obtaining details of medical history, compared to $11 \%$ of nurse questions. Nurses using CDSS frequently delivered questions designed as declarative statements requesting confirmation and which typically preferred a 'no problem' response. In contrast, GPs asked a higher proportion of interrogative questions designed to request information.

Conclusions: Nurses and GPs emphasise different aspects of the clinical assessment process during telephone triage. These different styles of triage have implications for the type of information available

\section{Strengths and limitations of this study}

- The first study to provide naturally occurring audio and video data directly comparing nurseled and general practitioner (GP)-led triage. It provides clear evidence that computer decision support software (CDSS) organises nurse questioning, creating very different triage interactions to GPs not using CDSS.

- This study was limited by the inclusion of only two GP practices. Given further training in the use of CDSS, the nurses in this study might also have conducted triage differently.

- The different styles of triage we observed have implications for the type of information collected from patients, and for how patients experience triage.

following nurse-led or doctor-led triage, and for how patients experience triage.

\section{INTRODUCTION}

Telephone triage is the process where calls from people with a healthcare problem are received, assessed and managed by giving advice or by referral to a more appropriate service. ${ }^{1}$ It is increasingly being used internationally to help with the provision of out-of-hours care, manage demand for care or provide an additional source of help and advice. $^{2}$ In the UK, the use of the telephone to triage patients requesting same-day appointments represents one strategy to manage the increasing workload taking place in primary care. ${ }^{3}$ Nurses and general practitioners (GPs) may provide telephone triage and consultation, with nurses typically trained to use computerised decision support software to provide this service, both in office hours and out of hours. While there is some 
evidence that telephone triage may reduce GP workload, ${ }^{45}$ there is equivocal evidence that telephone triage is a safe $e^{6-9}$ and satisfactory means of delivering care to patients. ${ }^{5} 10$ The quality of patient-clinician interaction during triage and telephone consultation is key to aspects of safety, effectiveness, patient experience ${ }^{211}$ and, potentially, to health outcomes. ${ }^{12}$ How clinicians communicate with patients and respond to their presenting concerns within telephone triage consultations is therefore central to decisions about its delivery within primary care.

Research comparing how GPs and nurses communicate with patients within face-to-face consultations has identified patterned differences in the process of assessing patients and in the opportunities afforded to patients to explain their presenting problems. ${ }^{13}$ Richards $e t a l^{14}$ used GPs and nurses to retrospectively assess audio-taped nurse-led telephone triage, and found that GPs and nurses had only moderate levels of agreement on the level of information sought by nurses and on the appropriateness of triage outcome. A recent study using conversation analytic methods to compare doctor-patient consultations conducted face-to-face and on the telephone ${ }^{15}$ found little difference in consultation styles between the two methods of delivering care, but found that patients differed in the number and complexity of topics introduced on the telephone compared with face-to-face consultations.

The role of computer decision support software (CDSS) and the professionals who use it is central to assessing the safety and effectiveness of telephone triage. In primary care, CDSS is promoted as supporting the clinical expertise of nurses to conduct triage, ${ }^{16}$ representing a substitute for the expertise provided by GPs who do not use CDSS to triage patients. While there is evidence that using CDSS to support clinical decisions is a safe and effective means of triaging patients, ${ }^{17}$ other research suggests that nurses orientate to potential dissonance between CDSS question prompts and the specific circumstances of the caller's concern $^{18}$; interact with the patient and the CDSS in 'purposive interaction chains ${ }^{19}$; or regularly deviate from and modify CDSS prompted questions, potentially leading to a divergence rather than standardisation in triage outcomes. ${ }^{20}$

A recent retrospective case review of closed malpractice claims regarding telephone-related consultations in the $\mathrm{USA}^{21}$ found that $38 \%$ of litigation cases were because of problems with communication. Reporting on cases involving clinicians from a range of professional disciplines, Katz et al suggested that as workload increases, clinicians may rush through triage and in some cases patients may be doing the triage rather than the clinician.

In a similar analysis of calls to Swedish Healthcare Direct, Ernesater $e t a l^{22}$ reported that failures in communication and asking the caller too few questions were commonly observed in malpractice claims, a finding also reported in a Dutch study of simulated calls to out-of-hours centres. ${ }^{17}$
If nurse/GP triage is to be widely used within primary care in the UK, there is therefore a need for greater insight into patient-clinician telephone communication that is both safe and acceptable to patients. Until now, there has been no research that has directly compared telephone triage communication of nurses using CDSS with GPs conducting telephone triage without the additional support of CDSS.

We compared communication between GP-led and nurse-led computer-supported telephone triage in primary care. We proposed to contribute to an understanding of the 'real-time' use of telephone triage in primary care by nurses and GPs. In this article, we focus on a key element of telephone triage-the interrogative series driving the process of clinical assessment-a crucial source of information to support decisionmaking about triage outcomes.

\section{PARTICIPANTS AND METHODS}

The research was a substudy that formed part of the recruitment process for the ESTEEM trial, ${ }^{23}$ the first multicentre randomised controlled trial to compare GP-led versus nurse-led telephone triage versus usual care for UK patients requesting same-day appointments. A qualitative comparative study of nurse-led and GP-led triage consultations was used to enable close analysis of interaction between clinicians and patients; and the role the CDSS played in organising nurse-patient interactions.

Five intervention practices were approached (2 nurses and 3 GPs), of which two were recruited successfully (1 nurse and 1 GP). Four nurses and four GPs participated. Participating practices were not systematically triaging patients prior to their inclusion in ESTEEM, and therefore nurses had to be trained in the use of CDSS prior to starting the trial. However, data for the substudy were not collected until practices were in their final week (average 8 weeks post-training in CDSS) of participation in ESTEEM. Patients (or their proxy) who phoned their surgery requesting a same-day, face-to-face appointment with a GP were eligible for participation. Patients were excluded from the substudy if they did not fulfil the criteria for inclusion to the main ESTEEM trial, ${ }^{23}$ including:

- Patients who were (1) too ill to participate; (2) unable to speak English; (3) temporary residents.

- Young people aged 12-15.9 years.

- Children under 12 years unless a proxy phoned on their behalf.

Over a 2-day period in each practice, 81 audiorecordings of telephone triage ( 47 nurse and $34 \mathrm{GP}$ ) and 35 video-recordings of nurses' use of Odyssey CDSS were made. Written consent was given by patients to analyse 51 complete recorded calls (22 nurse and 29 GP) including 10 video-recordings. Video-recordings were synchronised with audio-recordings to enable analysis of how nurses used Odyssey during triage. Demographic data collected as part of the ESTEEM trial were also extracted for 


\begin{tabular}{lll} 
Table 1 Call sample & & \\
\hline Call sample descriptors & Nurse-led & GP-led \\
\hline Male & 5 & 6 \\
Female & 17 & 23 \\
Mean patient age (years) & 45.2 & 44.7 \\
Patient age range (years) & $1.4-88.4$ & $0.2-80.6$ \\
Calling on own behalf & 17 & 22 \\
Calling on behalf of other & 5 & 7 \\
Unknown problem & 18 & 18 \\
Known problem & 5 & 11 \\
Single-issue problem & 22 & 23 \\
Multiple problems & 0 & 6 \\
\hline
\end{tabular}

consented patients. Call length was determined by the length of the recording, which began as soon as the patient picked up the telephone and ended at the call close. Forty women (17 nurse, 23 GP) and 11 men participated, with an average age of 44 years $(\mathrm{SD}=25$; see table 1). In addition, triage outcome was collected for all consenting patients (see table 2).

\section{Analysis}

All calls were transcribed in detail according to standard conversation analytic conventions ${ }^{24}$ (see table 3 for transcription key). Call lengths were measured from audiorecordings. This led to identifying potentially important differences between the two groups in call length and numbers of questions clinicians asked. In order to understand the nature of these differences, we adapted an established conversation-analytic coding scheme for analysis of question-response sequences. ${ }^{25}$ Conversation analysis is increasingly being used to support medical research aimed at understanding the distribution of interactional practices by offering operational definitions of phenomena that can subsequently be coded and counted. ${ }^{26}$ Inclusion criteria for the coding scheme were as follows:

- Questions had to effectively seek to elicit information, confirmation or agreement whether or not they made use of an interrogative sentence type;

- Questions seeking acknowledgement were not coded because they sought neither confirmation nor affirmation;

- Repair questions ('Pardon') as well as partial repeats ('He went where?') were not coded;

\begin{tabular}{llc} 
Table 2 Triage outcome & & \\
\hline Triage outcome & Nurse-led & GP-led \\
\hline Same day with nurse & 10 & 2 \\
Same day with GP & 6 & 18 \\
Nurse or GP next day & 3 & 0 \\
Nurse or GP 3-7 days & 1 & 1 \\
Self-care & 2 & 3 \\
Nurse or GP $>7$ days & 0 & 5 \\
\hline
\end{tabular}

\begin{tabular}{|c|c|}
\hline (.) & $\begin{array}{l}\text { A micropause, hearable but too short to } \\
\text { measure }\end{array}$ \\
\hline$>$ he said< & $\begin{array}{l}\text { 'Greater than' and 'lesser than' signs } \\
\text { enclose speeded-up talk. Occasionally they } \\
\text { are used the other way round for slower talk }\end{array}$ \\
\hline Underlining & $\begin{array}{l}\text { Indicates emphasis; the extent of } \\
\text { underlining within individual words locates } \\
\text { emphasis and also indicates how heavy it is }\end{array}$ \\
\hline$\uparrow \downarrow$ & $\begin{array}{l}\text { Vertical arrows precede marked pitch } \\
\text { movement, over and above normal rhythms } \\
\text { of speech. They are used for notable } \\
\text { changes in pitch beyond those represented } \\
\text { by stops, commas and question marks }\end{array}$ \\
\hline $\begin{array}{l}\text { she wa:: } \\
\text { nted }\end{array}$ & $\begin{array}{l}\text { Colons show degrees of elongation of the } \\
\text { prior sound; the more the colons, the more } \\
\text { the elongation }\end{array}$ \\
\hline [ ] & $\begin{array}{l}\text { Square brackets mark the start and end of } \\
\text { overlapping speech. They are aligned to } \\
\text { mark the precise position of overlap as in } \\
\text { the example below }\end{array}$ \\
\hline${ }^{\circ} \uparrow !$ know it, & $\begin{array}{l}\text { 'Degree' signs enclose hearably quieter } \\
\text { speech }\end{array}$ \\
\hline
\end{tabular}

- Questions that suggest, propose or offer something to another as well as questions that request something from another were not coded (eg, "Can I just confirm your date of birth before we go any further" or "Just bear with me a moment, we'll have a look for you").

The scheme included 12 coding categories (see box 1), including question design, the action they performed and the responses elicited. We also coded for an additional dimension of the broader activity questions were oriented towards. Working from the inclusion criteria with audio-recordings and transcripts, all eligible questions were identified and agreed on by JM, RB and JP $(n=484)$. Each of the two raters $(J M, R B)$ then independently coded $10 \%$ of questions to determine the inter-rater reliability. $\kappa$ Scores were calculated for all coding

Box 1 Question-response coding scheme (adapted from Stivers and Enfield 2010)

- Semantic structure

- Through-produced multiquestions

- Polar questions-turn-final element; negative marking; declarative

- Type of Q-word in content Wh-questions

- Social action of question

- Response type

- Response timing

- Confirming or disconfirming answer to polar question

- Form of answer to polar question

Added for analysis of triage interactions:

- Question activity-reported symptom; wider-information gathering; eliciting patient explanations, views; medical history 
categories revealing moderate-to-high levels of agreement (0.67-1.00). JM coded the remaining data and ambiguous cases were discussed in order to reach a consensus on the final code. JM, RB and JP then closely examined prototypical cases identified in the coding of questionresponse sequences and which demonstrated recurrent patterns of interaction across the consultations.

\section{RESULTS}

\section{Question activity}

Average call length (minutes:seconds-mean, SD, range) was similar across both arms, (nurse-led=4:39, 2:22, 1:4510:46; GP-led=4:37, 1:26, 1:29-8:14). However, nurses asked patients an average of $14.7(\mathrm{SD}=6.4$, range $=4-28)$ questions to assess the problem during telephone triage consultations, in contrast to only 5.5 ( $\mathrm{SD}=4.6$, range $=0$ 17) asked by GPs. Nurses were predominantly oriented towards two types of question activity (table 4)-the assessment of the patient's reported symptom (nurse $32.1 \%$; GP 21.9\%) and wider information gathering around the reported symptom (nurse $56.8 \%$; GP $32.5 \%$ ). In contrast, GP questions were more evenly distributed across four types of question activity, including eliciting the patient's own explanations for their symptoms (nurse $2.5 \%$; GP $13.1 \%$ ) -for example, There's no obvious explanation for that? and seeking a more detailed medical history from the patient (nurse 8.6\%; GP 30\%)-for example, What do you attend the hospital for?

\section{Question design and action}

Over three-quarters of questions deployed by GPs and nurses (nurse $82.1 \%$; GP 76.3\%) were polar questions. A key feature of polar questions is that they are designed for either a yes or no response. ${ }^{27}$ Second, polar questions are commonly employed in medical history-taking where they are frequently designed to prefer a 'no problem' response. For example, the inclusion of the negative polarity item 'at all' tilts, Has she a temperature at all? to prefer a no and therefore the absence of fever (an optimal state of affairs). In contrast, And you're breathing normally? prefers a yes response and therefore the absence of breathing problems (again an optimal state of affairs). GPs used a higher proportion of polar questions with an interrogative design (nurse 29.94\%; GP $46.25 \%$ )-for example, Have you vomited at all? while over half of the nurses' polar questions were designed as declarative statements (nurse 52.16\%; GP 30\%) with a presupposed answer embodied within the question for confirmation or disconfirmation-for example, passing urine okay? The proportion of polar, declarative questions used by nurses is also reflected in the proportion of questions that nurses asked that requested caller confirmation (nurse 53.7\%; GP 31.87\%). This stood out in

Table 4 Interrogative series: coding for question activity, design and action

\begin{tabular}{|c|c|c|}
\hline & Nurse (\%) & GP (\%) \\
\hline \multicolumn{3}{|l|}{ Activity } \\
\hline $\begin{array}{l}\text { Reported symptom } \\
\text { eg, Is he getting more breathless? }\end{array}$ & 32.1 & 21.9 \\
\hline $\begin{array}{l}\text { Wider information gathering } \\
\text { eg, and have you got a temperature at all do you feel hot? }\end{array}$ & 56.8 & 32.5 \\
\hline $\begin{array}{l}\text { Eliciting patient's concerns/ideas/expectations } \\
\text { eg. There's no obvious explanation for that? }\end{array}$ & 2.5 & 13.1 \\
\hline $\begin{array}{l}\text { Medical history } \\
\text { eg, And is there any family history of arthritis of any sort? } \\
\text { Design }\end{array}$ & 8.6 & 30.0 \\
\hline $\begin{array}{l}\text { Polar questions-interrogative } \\
\text { eg, Has she vomited at all } \\
\text { (Reported symptom and wider-info gathering activities only) }\end{array}$ & 29.9 (25.9) & $46.3(26.3)$ \\
\hline $\begin{array}{l}\text { Polar questions-declarative } \\
\text { eg, And she's weeing okay } \\
\text { (Reported symptom and wider-info gathering activities only) }\end{array}$ & $52.2(45.4)$ & $30.0(12.5)$ \\
\hline $\begin{array}{l}\text { Content } \mathrm{WH}-\text { questions } \\
\text { eg, And when did the tiredness first start }\end{array}$ & 13.3 & 18.8 \\
\hline $\begin{array}{l}\text { Alternative questions } \\
\text { eg, Is it quite bad or just a little bit of dizziness } \\
\text { Action }\end{array}$ & 4.6 & 5.0 \\
\hline $\begin{array}{l}\text { Request for confirmation } \\
\text { eg, And you said no discharge and no pain } \\
\text { (Reported symptom and wider-info gathering activities only) }\end{array}$ & $53.7(46.6)$ & 31.9 (13.8) \\
\hline $\begin{array}{l}\text { Request for information } \\
\text { eg, Do you wear glasses or contact lenses? } \\
\text { (Reported symptom and wider-info gathering activities only) }\end{array}$ & $46.0(42.3)$ & $68.1(40.6)$ \\
\hline
\end{tabular}


contrast to GPs predominantly asking questions that requested information rather than confirmation (nurse $45.99 \%$; GP $68.13 \%$ ).

Nurses using CDSS were therefore deploying a higher proportion of declarative questions about the patient's reported symptom or wider information gathering than GPs, designed to optimise the report of the patient's situation by ruling out a variety of medical problems (nurse $45.37 \%$; GP $12.51 \%$ ). In contrast, GPs, not using CDSS and therefore more able to self-determine their questions, employed questions more evenly distributed across the four different activities, typically designed as interrogatives aimed at requesting information from the caller. In order to reveal the implications of these different distributions for how GPs and nurses, using CDSS, conducted telephone triage in our sample, it is necessary to examine how these differences in question number, activity, design and action are consequential within the triage interactions themselves.

\section{Gathering information during nurse-led triage:}

\section{a 'no problem' question series}

When patients present a problem to clinicians, they may report several symptoms. Nurses, using Odyssey CDSS, need to select one of these symptoms and enter a key word to activate the CDSS for conducting triage with the patient. This activates a pop-up box with a series of symptom-related questions that prompt the nurse to ask of the patient. The nurse can select which questions to ask first, but it is important that the nurse asks those with a red or orange flag positioned adjacent to the question. Red-flagged questions have a default setting at the highest level of urgency and therefore, if left unanswered, Odyssey will recommend an emergency response. For each question asked, Odyssey prompts a set of responses in an additional pop-up box from which the nurse must select one. Odyssey therefore imposes an organisational structure on nurse questioning that is absent from GP-led triage. This structure is not only in terms of the number and order in which questions are asked, but also how questions are designed to elicit a response from patients that fits those offered by Odyssey.

The sequence and screenshot in box 2 (figure 1) illustrates an interrogative series common to the wider information gathering activity in nurse consultations, using polar questions which first constrain the type of response available to patients and second are designed to prefer 'no problem' type responses. In this sequence, the caller is a mother of an infant who has a high temperature. The nurse has activated the CDSS using the keyword 'high temperature', which has led to the CDSS prompting the nurse to ask the caller about vomiting. This first question is a fully formed interrogative, with the negative polarity item 'at all' preferring a no response, indicating the nurse presupposing an absence (and therefore 'no problem') rather than the presence of vomiting. The preferred response is therefore one that should not only be brief (yes/no) but one that also

\section{Box 2 Nurse-led triage: 'no problem' design questions}

$\mathrm{N}$ : And has she vomited at a:ll

$P$ : No (.) she hasn't no

$\mathrm{N}$ : And $>$ I know you ha-< you said you hadn't given her her a $>$ bottle this morni-< but she is taki::ng (.) flui::ds

$P$ : Yes she's drinking some (water)

$\mathrm{N}$ : And she's weeing okay

$P$ : Yeah she's weeing absolutely fine

$\mathrm{N}$ : No ra::sh?

$P$ : No no ra:sh

N: 0个ka::y, (1.8) and no other symptoms she's not coughi::ng

$P$ : $\uparrow$ She had a little cough this morning (3.2) but not a constant cou:gh

$\mathrm{N}$ : $\uparrow$ And $\uparrow$ normally we:ll no other problems normally::

$P$ : No she's normally fine running arou:nd like a,

At $1 \mathrm{~min}, 45 \mathrm{~s}$ *

* The times here refer to the time that the extract begins in relation to the start of recording.

informs the nurse of the absence rather than presence of vomiting, which will enable a quick transition to the next topic. The mother offers the preferred response with a partial repeat of the nurse's question 'No, she hasn't'. The nurse is then able to move on to the next item in the list of prompted questions, 'but she is taking fluids'. This time the nurse's question is designed as a declarative with an optimised presupposition aimed at obtaining confirmation. The mother again responds with a short reiteration of the nurse's statement, functioning to confirm that the nurse's presupposition is correct 'Yes she's drinking some water'. The same question design is then repeated 'and she's weeing okay'.

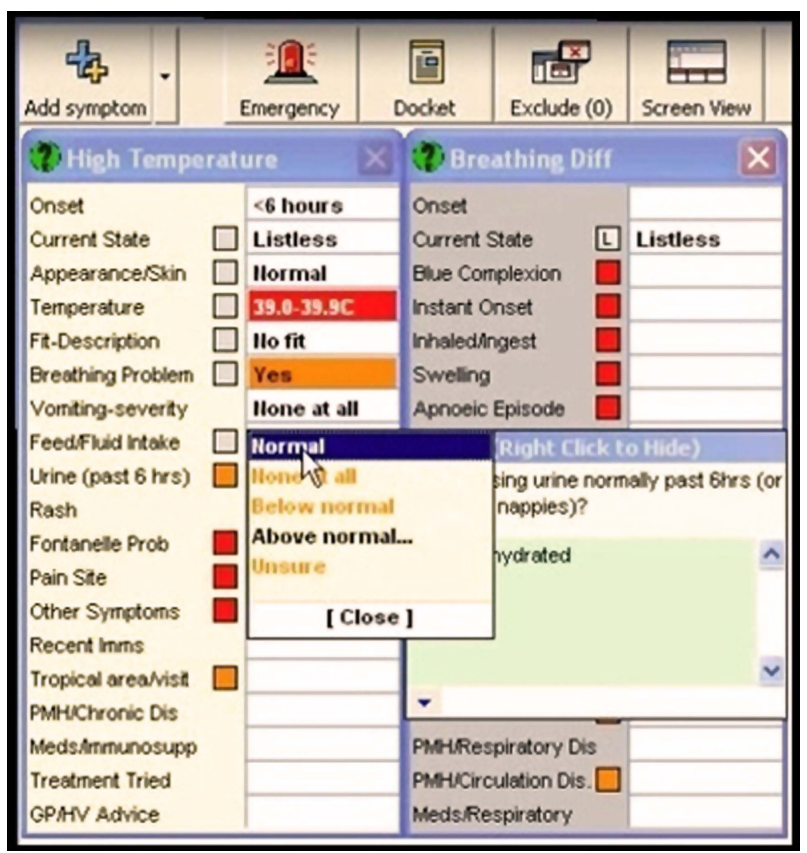

Figure 1 Nurse-led triage: CDSS during 'no problem' question series. 
Again the mother repeats the nurse's words and confirms 'Yes she's weeing absolutely fine'. The nurse then switches to a negative declarative design presupposing an absence of a rash, and again we can see the mother follows the same pattern. However, following the nurse's next question, we see a slight variation in the mother's response. The nurse again presents a negative declarative question 'she's not coughing', but this time the patient appears to disconfirm the nurse's presupposition. However, this is not a direct disconfirmation (eg, Yes she is coughing), but instead the mother qualifies her response-not a constant cough, functioning to uphold the nurse's presupposition as at least partially correct. Finally, the negative declarative 'no other problems normally' is issued with the mother repeating the previous pattern, confirming the optimised presupposition that there are 'no other problems.'

This example was typical of how nurses could be seen to manage the demands of the CDSS prompts by adapting questions that enabled swift progression through the consultation. The use of And to preface questions, use of multiple declaratives, optimised for confirmation, and a process of ellipsis whereby questions were shortened made this information gathering activity appear more as a checklist designed to rule out serious difficulties rather than orienting to the patient's specific presenting problem. Consequently, contracted sequences of interactions with short turns, illustrated in box 2 , were a common feature in the nurse-led data but virtually absent in the GP-led data.

In a few cases, nurses could be seen to incorrectly presume a 'no problem' response from patients, resulting in some interactional difficulty whereby patients had to disconfirm the presupposition embodied in the question. In box 3, an example is provided of a nurse asking about the radiation of pain, delivered as an optimised negative declarative statement for the patient to confirm. However, there is a pause and then a nonstraightforward response from the patient 'We::ll...', which serves to neither confirm nor disconfirm the nurse's presupposition. Even after referring to a previous statement and restating that there is some pain in the buttocks, the patient softens the weight of her disconfirmation with a further 'I don't know.' The effect is that the 'no problem' design in this case leads to a lack of

Box 3 Nurse-led triage: No problem design causing interactional difficulty

$\mathrm{N}$ : And you don't feel that it's moving down into the buttocks (0.6)

P: We:.Il [l'd say it] (0.6) well I don't kno:...: it=

$\mathrm{N}: \quad[(?)]$

P: $=($.$) it's as I sa:y it ha- halfway do::wn the buttock an- (.) not$ so far in I would think I don't $\uparrow$ know

$\mathrm{N}$ : Okay that's fi::ne

At $1 \mathrm{~min}, 53 \mathrm{~s}$ clarity as to the location of the patient's presenting symptoms, which is likely to be reflected in how the nurse records the patient's response in Odyssey.

\section{GP-led triage: questions designed to elicit patient's explanations}

In contrast to the exhaustive CDSS-led questioning about the nature of the reported symptom and wider information gathering around it apparent within the nurse-triage data, GPs were frequently observed eliciting the patient's explanations for their symptoms. In the extract outlined in box 4, it is evident that early on in the triage consultation, and immediately following the patient's reported symptom, the GP attempts to rule out any obvious explanations from the patient. Following an initial marked confirmation, the patient takes this opportunity to list the candidate explanations she has considered and the GP then moves straight to triage resolution. Importantly, despite the GP using polar declarative questions that request confirmation, they are orientated to the ongoing talk and caller's responses rather than presented as a series of checklist-style questions.

Interactions where the patient's explanations were oriented to and followed up by the GP were very rare in the nurse triage data and indicate GPs and nurses orientating to different aspects of the assessment process during triage. Box 4 provides an example of the potential of orienting to the patient's explanations for determining triage outcome more efficiently, without an exhaustive interrogative series delivered through the use of the CDSS. However, we observed many instances of GPs and patients engaging in lengthy discussions about the possible causes of symptoms as well as GPs obtaining a detailed wider history from the patient, both of which

Box 4 General practitioner-led triage: eliciting patient explanations

$P:=$ on it woke up this morning and my who::le (.) my whole eye and the whole left hand side of my face is complete swollen, my $\uparrow$ eyes almost closed,

$\mathrm{D}:{ }^{\circ} O \mathrm{kay}^{\circ}($.) and there's no obvious explanation for that $\mathrm{y}$ -

P: Not at all

D: ${ }^{\circ}$ No okay ${ }^{\circ}$

P: No I haven't I haven't suffered it, (.) y- y::0u know >l don't suffer with< hay feve:r (.) haven't been anywhe::re (.) unusual or $y$ - y- you kno::w didn't [walk the dogs in any (field)]

D: [(?) ] (o:r) or got anything in the eye or anything like that

P: No: (>got to admit<) I went to be:d at half past ten (.) perfectly fi::ne (.) and $\mathrm{m}-\mathrm{I}$ do sleep with my window slightly open

D: ${ }^{\circ}{ }^{\circ}{ }^{\circ}{ }^{\circ}$

P: Um a::nd (04.) went straight to sleep within an hour I just sat bolt upright (0.4) with >this like< $\uparrow$ burning sensa $\uparrow$ tion,

D: Okay (.) I think we're gonna need to take a look at you .hh can you [come] in this morning?

At $0 \mathrm{~min}, 14 \mathrm{~s}$ 
were less common in the nurse data. By contrast, nurses using CDSS did not engage in lengthy discussions about possible diagnoses even when one was offered by the patient, instead confining to the information gathering demands of the CDSS.

\section{Nurse-led triage without CDSS}

On a few occasions, the Odyssey software was not activated during the recorded nurse-led calls. In these cases, question-response sequences were identified that did not follow the same pattern as those where CDSS was in use. Box 5 provides an example of a sequence where the nurse, not using CDSS, begins the wider information gathering activity with a typical opening question about duration followed by a declarative question designed to confirm previous patient information. However, instead of then asking a series of questions aimed at gathering information on related symptoms, the nurse proposes a candidate diagnosis. In a similar way to the GP data, this potential diagnosis is then negotiated with the patient within the ongoing interaction.

\section{DISCUSSION}

Despite nurses asking three times as many questions as GPs, the similarity in the duration of triage calls is explained by the content and form of questions used and their different interactional consequences. Most notably, nurses' frequent use of 'no-problem' polar declaratives requesting confirmation, deployed predominantly to gather information around the reported symptom, created contracted question-response sequences. These features were almost completely absent in the GP data where more polar interrogatives were employed to request information from patients, taking a more unknowing stance and allowing more room for elaboration or sequence expansion. ${ }^{27}$ GP questions also launched a wider range of activities including eliciting patients' own views and obtaining a relevant medical history. This differential distribution of question design, action and activities in the GP data led to typically longer patient responses and subsequently to other kinds of practitioner contribution such as GP's own evaluations, patient education or advice. The nurses, using CDSS, and GPs, self-determining their questions, could therefore be considered to promote different aspects of

Box 5 Nurse offering diagnosis when not using computer decision support software

$\mathrm{N}$ : an ongoing, (.) she's had a (.) recent viral illness

P: Yeah

$\mathrm{N}$ : And she's pulling at her (0.6) ea::r is $\uparrow$ it?

P: Yea::h, (.) both of them

$\mathrm{N}$ : Is she [teething]?

At $1 \mathrm{~min}, 3 \mathrm{~s}$ the clinical assessment process during telephone triage. While nurses' focus on current symptoms emphasised an assessment of urgency and efficient risk management of the patient, GP's attempts to elicit patient's own explanations and obtain a wider medical history demonstrated more familiar consultation behaviours. ${ }^{28}$

The interactional differences observed in our sample reveal that nurse-led telephone triage, using CDSS, is not a straightforward like-for-like substitution for GP-led triage. The design of nurse questions could be seen as an adaptive strategy to the constraints of the software. As a result, their attempts to rule out more serious conditions manifested as a series of linked checklist-style questions that appeared closer to a social survey than a medical interaction orientated to patient's specific problems. This is reflected in the findings of the ESTEEM process evaluation (JL Campbell, personal communication, 2013), which found that some patients did not understand why nurses asked so many questions during triage calls, revealing that patients experience triage differently when conducted by a nurse using CDSS or a GP without CDSS.

Our findings also demonstrate that GPs acting independently, and nurses using CDSS, are likely to obtain different types of information from patients. The differences in information collected may have an impact on how GPs and nurses decide on management and disposition, and also on how patients are assessed in any subsequent consultation following the triage call. A key task for assessing the value of these different approaches therefore lies in research that examines how the content of triage calls is used in, or informs, subsequent face-to-face or other primary care consultations.

GPs have historically been cast as expert decision makers, and so it is perhaps not surprising that their eliciting of patient perspectives and detailed history have been identified as common features of both face-to-face and telephone consultations. ${ }^{15}$ Patient-centred consultation styles have been shown to lead to increased patient satisfaction, treatment adherence and treatment outcomes, ${ }^{29-33}$ and it would therefore seem to be a logical style to reproduce within triage calls. However, the primary aim of telephone triage is to manage and direct patients within the healthcare system. GPs, when eliciting patient explanations, may therefore contribute to longer triage times than might be necessary to determine the triage outcome. Nurses using CDSS, by contrast, would appear to strictly adhere to the end point of patient management and perhaps more efficiently determine the route patients should take in primary care. However, using CDSS involves extensive questioning, which may also unnecessarily contribute to longer triage times. How these different triage methods affect triage outcome and overall consultation time; whether training nurses to adapt their question design when using CDSS affects triage outcome; and how patients experience and respond to these different approaches are key issues requiring investigation. 
Limitations and strengths of the study

This study was limited by the inclusion of only two GP practices. It is possible that nurses and GPs conducting triage in other GP surgeries would have employed different patterns of distribution of question designs, actions and activities to those reported here. Although GPs did not use CDSS to triage patients, we also recognise that GPs may have actively consulted electronic patient records while triaging, which might have provided an interesting comparison to the nurse data.

Given further training and experience of the CDSS, nurses might have delivered different interactions from those we observed here. However, our findings clearly demonstrate how the CDSS imposes a structure on the number, order and design of nurse's questioning compared with GPs questioning. This is backed up by our observation of nurses' different questioning pattern when not using CDSS. Investigating how nurses with extensive training in the use of CDSS communicate with patients, and how this compares with nurses not using CDSS would therefore provide important insights into the contribution of CDSS in supporting nurses to deliver safe and effective patient management. The resonance between nurses' questioning in our data and interactions observed in National Health Service (NHS) Direct consultations ${ }^{18}$; and the GP's questioning style in our data and previous research on telephone consultations ${ }^{15}$ also indicates how our findings may be transferred to other primary care settings. Training for telephone triage could be designed to incorporate working with sample recordings and transcripts of real calls to illustrate the full range of questions that can be asked in the interrogative series; and how question design itself can be consequential for the nature of a patient's response. Studies such as the one reported here therefore offer important insights into the actual implementation of telephone triage using different professionals, and how CDSS can organise telephone triage interactions and patient experiences. Such insights can assist with the training of those professionals in conducting triage; with revealing how the design of CDSS systems might be more effectively configured; and with the management of patient expectations around new technologies for medical service delivery.

Although the parent trial to this study examined the issue of patient safety alongside telephone triage, ${ }^{23}$ we did not specifically examine safety in this qualitative study. Previous relevant reviews ${ }^{2} 34$ and individual studies $^{4} 61735$ are conflicting in respect of patient safety outcomes and the related matters of hospital admissions or A\&E attendance associated with triage. Specific concerns have been raised in relation to the quality of information gathering in telephone triage consultations, ${ }^{8} 17$ and the differences in information gathering between nurses using CDSS, and GPs not using CDSS, in our findings place communication, information gathering and the role of CDSS at the heart of ongoing debates about patient safety.

\section{Conclusion}

Our data suggest that nurse triage using CDSS is not a straightforward substitution for GP triage without CDSS. CDSS, employing algorithms designed to minimise risk, plays a fundamental role in organising nurse's questioning during triage leading to differences in the number, content and form of questions used by GPs and nurses. These differences have consequences for the type of information collected from patients during triage calls and for how patients experience those calls. These findings are based on a small sample, and it is not known how these triage styles are linked to triage outcomes. However, given the well-established relationship between consultation style and outcomes in primary care, our findings provide important evidence for the training of staff and for the design of CDSS in supporting staff to conduct telephone triage.

Acknowledgements The authors would like to thank all patients, nurses and GPs for participating in this study.

Contributors JM and RB led the development of the original protocol, funding application and coordinated the overall running of the project; and they also conducted the analyses, contributed to the interpretation of the findings and drafted and edited the paper. JP implemented the project protocol, including recruiting GP practice staff and patients and collection and storage of data. She contributed to the analyses, interpretation of findings and edited the paper. JLC contributed to the development of the original protocol, funding application and oversaw how the substudy was embedded within the running of the ESTEEM trial. He also contributed to the interpretation of the findings and edited the paper. VL contributed to the development of the original protocol and funding application. She also contributed to how the substudy was embedded within the running of the ESTEEM trial, and also to the interpretation of the findings and edited the paper. EF contributed to the development of the original protocol, funding application and obtained R\&D and ethical approval. She managed the implementation of the substudy within the running of the ESTEEM trial. She contributed to the interpretation of the findings and edited the paper.

Funding The study was funded by the South West GP Trust. The study was a substudy to the ESTEEM trial. ESTEEM was funded by the NIHR Health Technology Assessment programme (project number 08/53/15). The ESTEEM study is currently in press and will be published in full in the Health Technology Assessment Journal on the NIHR Journals Library website. Please see the NETSCC website for further project information.

\section{Competing interests None.}

Ethics approval The study was approved by the South West Research Ethics Committee, reference number: 09/H0202/53. All healthcare professionals and patients provided written consent before taking part.

Provenance and peer review Not commissioned; externally peer reviewed.

Data sharing statement No additional data are available.

Open Access This is an Open Access article distributed in accordance with the Creative Commons Attribution Non Commercial (CC BY-NC 3.0) license, which permits others to distribute, remix, adapt, build upon this work noncommercially, and license their derivative works on different terms, provided the original work is properly cited and the use is non-commercial. See: http:// creativecommons.org/licenses/by-nc/3.0/

\section{REFERENCES}

1. Lattimer V, George S. Nurse telephone triage in out-of-hours primary care. Prim Care Manage 1996;6:3-6.

2. Bunn F, Byrne G, Kendall S. The effects of telephone consultation and triage on healthcare use and patient satisfaction: a systematic review. Br J Gen Pract 2005;55:956-61. 
3. Salisbury C, Montgomery AA, Simons L, et al. Impact of advanced access on access, workload, and continuity: controlled before-and-after and simulated-patient study. $\mathrm{Br} J$ Gen Pract 2007;57:608-14.

4. Richards DA, Meakins J, Tawfik J, et al. Nurse telephone triage for same day appointments in general practice: multiple interrupted time series trial of effect on workload and costs. BMJ 2002;325:1214-17.

5. Jiwa M, Mathers NJ, Campbell MJ. The effect of GP telephone triage on numbers seeking same-day appointments. $\mathrm{Br} J$ Gen Pract 2002;52:390-1.

6. Lattimer V, George S, Thompson F, et al. Safety and effectiveness of nurse telephone consultation in out of hours primary care: randomised controlled trial. The South Wiltshire Out of Hours Project (SWOOP) Group. BMJ 1998;317:1054-9.

7. Holmstrom I. Decision aid software programs in telenursing: not used as intended? Experiences of Swedish telenurses. Nurs Health Sci 2007:9:23-8.

8. McKinstry B, Hammersley V, Burton C, et al. The quality, safety and content of telephone and face-to-face consultations: a comparative study. Qual Saf Health Care 2010;19:298-303.

9. Poole SR, Schmitt BD, Carruth T, et al. After-hours telephone coverage: the application of an area-wide telephone triage and advice system for paediatric practices. Paediatrics 1993;92:670-9.

10. McKinstry B, Walker J, Campbell C, et al. Telephone consultations to manage requests for same-day appointments: a randomised controlled trial in two practices. Br J Gen Pract 2002;52:306-10.

11. Roing M, Rosenqvist U, Holmstrom IK. Threats to patient safety in telenursing as revealed in Swedish telenurses' reflections on their dialogues. Scand J Caring Sci 2013;27:969-76.

12. Street RL Jr, Makoul G, Arora NK, et al. How does communication heal? Pathways linking clinician-patient communication to health outcomes. Patient Educ Couns 2009;74:295-301.

13. Collins S. Explanations in consultations: the combined effectiveness of doctors' and nurses' communication with patients. Med Educ 2005;39:785-96.

14. Richards DA, Meakins J, Tawfik J, et al. Quality monitoring of nurse telephone triage: pilot study. J Adv Nurs 2004;47:551-60.

15. Hewitt $\mathrm{H}$, Gafaranga J, McKinstry B. Comparison of face-to-face and telephone consultations in primary care: qualitative analysis. $\mathrm{Br} \mathrm{J}$ Gen Pract 2010;60:201-12.

16. Plain Healthcare. Odyssey TeleAssess. 2013. Retrieved July 2013. http://www.plainhealthcare.co.uk/our-products/odyssey-range/ teleassess

17. Derkx HP, Rethans J-JE, Muijtiens AM, et al. Quality of clinical aspects of call handling at Dutch out of hours centres: cross sectional national study. BMJ 2008;337:1264.

18. Pooler J. Technology and talk in calls to NHS direct [PhD]. Loughborough University, 2010.

19. May CR, Mair F, Finch T, et al. Development of a theory of implementation and integration: normalization process theory. Implement Sci 2009;4:29.
20. Greatbatch D, Hanlon G, Goode J, et al. Telephone triage, expert systems and clinical expertise. Soc Health IIIn 2005;27:802-30.

21. Katz HP, Kaltsounis D, Halloran L, et al. Patient safety and telephone medicine: some lessons from closed claim case review. J Gen Intern Med 2007;23:517-22.

22. Ernesäter A, Winblad U, Engström M, et al. Malpractice claims regarding calls to Swedish telephone advice nursing: what went wrong and why? J Telemed Telecare 2012;18:379-83.

23. Campbell JL, Britten N, Green C, et al. The effectiveness and cost effectiveness of telephone triage of patients requesting same day consultations in general practice: a cluster randomised controlled trial comparing nurse-led and GP-led management systems (ESTEEM). Trials 2013;14:4. http://www.trialsjournal.com/content/ $14 / 1 / 4$

24. Jefferson $\mathrm{G}$. A glossary of transcript symbols with an introduction. In: Lerner G, ed. Conversation analysis: studies from the first generation. Philadelphia: John Benjamins, 2004:13-31.

25. Stivers T, Enfield NJ. A coding scheme for question-response sequences in conversation. J Pragmatics 2010; 42:2620-6.

26. Gill VT, Roberts F. Conversation analysis in medicine. In: Sidnell J Stivers T, eds. The handbook of conversation analysis. Oxford: Wiley-Blackwell, 2013:513-41.

27. Heritage J. Questioning in medicine. In: Freed AF, Ehrlich S, eds Why do you ask?: The function of questions in institutional discourse. New York: Oxford University Press, 2009:42-68.

28. Heritage J, Maynard DW. Introduction: analyzing interaction between doctors and patients in primary care encounters. In: Heritage $\mathrm{J}$, Maynard D, eds. Communication in medical care: interaction between primary care physicians and patients. Cambridge: Cambridge University Press, 2006:1-21.

29. Bertakis D, Roter D, Putnam SM. The relationship of physician medical interview style to patient satisfaction. J Fam Pract 1991;32:175-81.

30. Little $\mathrm{P}$, Everitt $\mathrm{H}$, Williamson I, et al. Observational study of effect of patient centredness and positive approach on outcomes of general practice consultations. BMJ 2001;323:908-11.

31. Roter DL, Hall JA, Katz NR. Relations between physicians' behaviours and analogue patients' satisfaction, recall and impressions. Med Care 1987;25:437-51.

32. Roter DL, Hall JA, Kern DE, et al. Improving physicians' interviewing skills and reducing patients' emotional distress: a randomized clinical trial. Arch Intern Med 1995;155:1877-84.

33. Stewart M. What is a successful doctorpatient interview? A study of interactions and outcomes. Soc Sci Med 1984;19:167-75.

34. Huibers L, Smits M, Renaud V, et al. Safety of telephone triage in out-of-hours care: a systematic review. Scand J Prim Health Care 2011;29:198-209.

35. Thompson F, George S, Lattimer V, et al. Overnight calls in primary care: randomised controlled trial of management using nurse telephone consultation. BMJ 1999;319:1408. 